\title{
Artifacts in spine magnetic resonance imaging due to different intervertebral test spacers: an in vitro evaluation of magnesium versus titanium and carbon-fiber-reinforced polymers as biomaterials
}

\author{
Thorsten Ernstberger • Gottfried Buchhorn • \\ Gabert Heidrich \\ Received: 19 April 2009 / Accepted: 12 May 2009/Published online: 26 May 2009 \\ (C) Springer-Verlag 2009
}

\begin{abstract}
Introduction Intervertebral spacers are made of different materials, which can affect the postfusion magnetic imaging (MRI) scans. Susceptibility artifacts especially for metallic implants can decrease the image quality. This study aimed to determine whether magnesium as a lightweight and biocompatible metal is suitable as a biomaterial for spinal implants based on its MRI artifacting behavior.

Materials and methods To compare artifacting behaviors, we implanted into one porcine cadaveric spine different test spacers made of magnesium, titanium, and carbon-fiberreinforced polymers (CFRP). All test spacers were scanned using two T1-TSE MRI sequences. The artifact dimensions were traced on all scans and statistically analyzed.

Results The total artifact volume and median artifact area of the titanium spacers were statistically significantly larger than magnesium spacers $(p<0.001)$, while magnesium and CFRP spacers produced almost identical artifacting behaviors $(p>0.05)$.
\end{abstract}

\footnotetext{
T. Ernstberger $(\triangle)$

Center for Spinal Surgery, Klinikum Bad Bramstedt,

Oskar-Alexander-Straße 26,

24576 Bad Bramstedt, Germany

e-mail: ernstberger@klinikumbb.de

G. Buchhorn

Biomaterial Laboratory, Department of Orthopedic Surgery,

University of Gottingen,

Robert-Koch-Straße 40,

37075 Göttingen, Germany

G. Heidrich

Department of Diagnostic Radiology, University of Gottingen,

Robert-Koch-Straße 40,

37075 Göttingen, Germany
}

Conclusion Our results suggest that spinal implants made with magnesium alloys will behave more like CFRP devices in MRI scans. Given its osseoconductive potential as a metal, implant alloys made with magnesium would combine the advantages to the two principal spacer materials currently used but without their limitations, at least in terms of MRI artifacting.

Keywords Magnesium alloys · Innovative biomaterials . Interbody test implants $\cdot$ MRI artifacting

\section{Introduction}

Spinal fusion devices such as implantable interbody spacers are well-established and routinely used by spine surgeons to keep adjacent vertebrae spaced apart while bone ingrowth and fusion take place. Such spacers also provide weightbearing support between adjacent vertebrae. In this context, titanium alloys as well as nonmetal materials like carbonfiber-reinforced polymers (CFRP) are commonly used [1, 3, $8,11,14,17,21]$. These biomaterials have enjoyed clinical success and rapid widespread use by improving patient outcomes.

However, these materials have clinical and radiological limitations. Titanium is an excellently bioinert material that exhibits high biocompatibility. Titanium spacers produce good bone ingrowth without bone grafting. However, in magnetic resonance imaging (MRI) studies, titanium-based implants tend to cause distortion of the magnetic field which may obscure normal regional anatomy. These properties pose difficulties in the postoperative MRI follow-up and evaluation of the fusion process due to the artifacting it causes $[4,6,7,12,15]$. 
The other principal material used for spacers consists of CFRP. Spacers made of this nonmetallic biomaterial are not associated with the postoperative diagnostic problems of titanium because carbon produces a very low rate of artifact reactions which allows easier evaluation of the fusion process by MRI [4]. Carbon's modulus of elasticity affords good load bearing with sufficient hardness. But unlike titanium, carbon spacers undergo poor osteointegration because a soft tissue interface develops around the material surface that prevents direct ingrowth of bone. As a result, carbon spacers have to be filled with bone allografts to achieve long-term stability $[1,4]$. CFRP implants have therefore been reviewed very critically in the literature [17].

Surgeons, over a century ago, recognized the potential of the lightweight metal magnesium as a biocompatible, osteoconductive, and degradable implant material [9]. In 1907, Lambotte was the first to introduce magnesium-based orthopedic devices; using a pure magnesium plate, he secured a bone fracture of the lower leg with gold-plated nails [9]. A half a century later, magnesium-based metals were reported to have osteoconductive bioactivity and produce a more
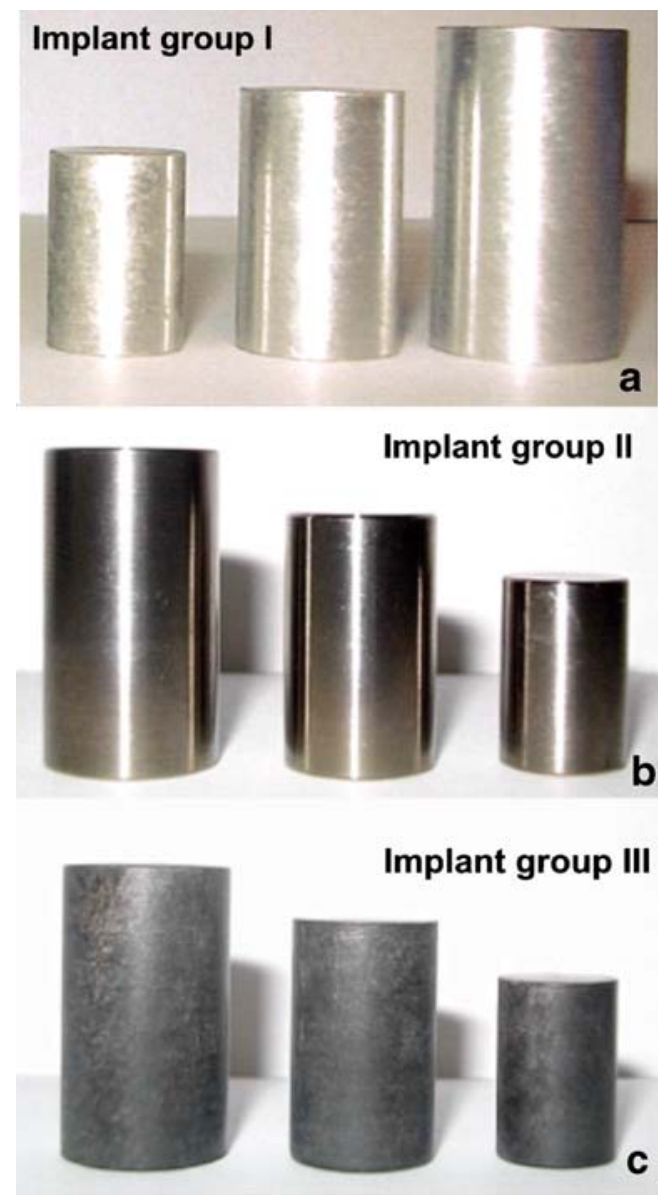

Fig. 1 a-c Cylindrical EST implants. a Magnesium (implant group I). b Titanium (implant group II). c CFRP (implant group III) rapid formation of hard callus when used to support fractures in humans [20, 27]. The large amount of evidence supporting the clinical advantages of magnesium has been summarized in a recent review paper [18]. None of the studies to date have yet investigated the diagnostic behavior of magnesium in MRI. This situation motivated us to determine whether magnesium is a suitable biomaterial for spinal implants by studying its MRI artifacting behavior.

\section{Material and methods}

To evaluate the behavior of spacers made with a magnesium alloy, we compared their artifacting in diagnostic MRI scans with that of spacers made of a conventional titanium alloy and of CFRP. We consecutively implanted three spacers made of each of the three biomaterials dimensioned in small, medium, and large sizes in one cadaveric spine of a Gottingen minipig (Figs. 1a-c and 2). The three spacers in group I were made of a magnesium-aluminum-manganese alloy (MgAlMn50), the three in group II of a titaniumaluminum-vanadium alloy (TiAl6V4), and those in group III of a CFRP.

Table 1 presents the implant characteristics. A cylinder was chosen as for spacer shape because cylinders have demonstrated lowest rate of MRI artifacting behavior [4].

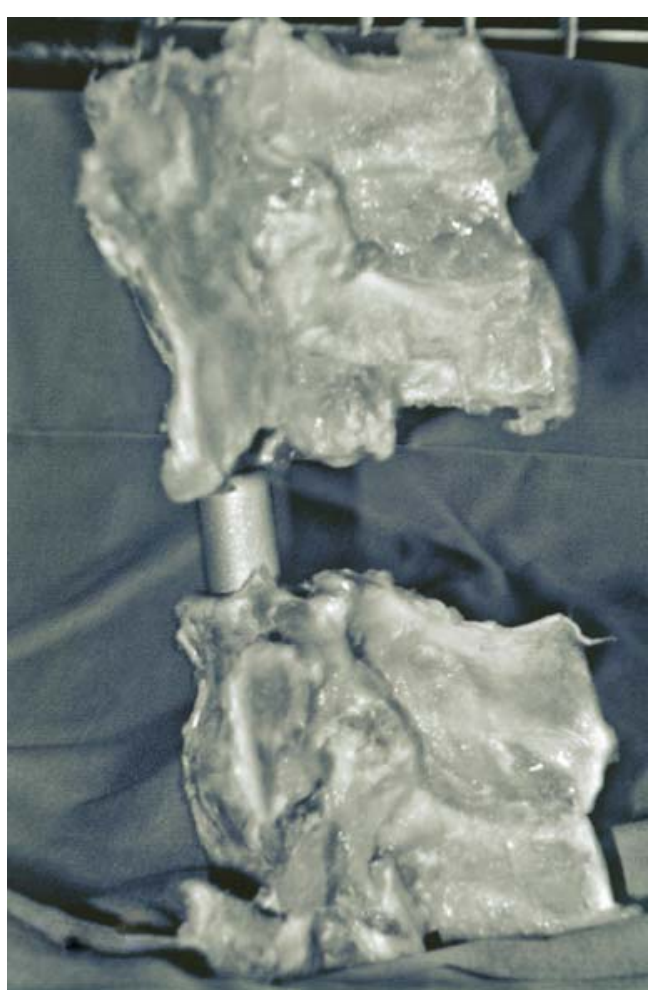

Fig. 2 Cadaveric porcine spine model with an implanted medium titanium test cylinder 
Table 1 Spacer dimensions.

\begin{tabular}{lccc}
\hline $\begin{array}{l}\text { Sizes for } \\
\text { all groups }\end{array}$ & $\begin{array}{l}\text { Dimensions height } \times \\
\text { base area, } \mathrm{cm} \times \mathrm{cm}^{2}\end{array}$ & $\begin{array}{l}\text { Cross-sectional } \\
\text { area (CSA), } \mathrm{cm}^{2}\end{array}$ & $\begin{array}{l}\text { Implant } \\
\text { volume (IV), } \\
\mathrm{cm}^{3}\end{array}$ \\
\hline Small & $1.5 \times 0.78$ & 1.5 & 1.2 \\
Medium & $2.0 \times 1.13$ & 2.4 & 2.3 \\
Large & $2.5 \times 1.54$ & 3.8 & 3.5 \\
\hline
\end{tabular}

The spacer sizes - small, medium, and large - were dimensioned the same for each group (height in centimeter $\times$ base area in square centimeter); and their implant volume (IV) in cubic centimeter and cross-sectional area (CSA) in square centimeter were calculated for each size (Table 1). The spacer sizes were dimensioned as listed after a Newman-Keuls multiple-comparison analysis showing that the selected sizes would produce significantly different artifacting behaviors $(p<0.001)$. Thus, a total of nine individual spacers were implanted, scanned by MRI, and evaluated for their artifacting behavior on the scans.

Spacer implantation

For each serial MRI study, the cylindrical implant was placed exactly between two adjacent vertebrae of the cadaveric porcine spine. The spine with implant was then completely packed in a soft tissue mass and placed in a plastic container [5]. To create comparable trial conditions, markings were drawn on the container wall to demarcate the vertebrae and implant positions. These demarcations were used to define the median artifact area (MAA). The container with the spine implanted with each spacer was examined by serial MRI.

Fig. 3 a-c Median MRI artifact range depicted in a selection of three large test implants
Magnetic resonance imaging

MRI was performed with a 1.5-T MRI (Magnetom Symphony, Siemens AG Medical Solutions, Erlangen, Germany). The T1w-TSE sequences were used to acquire a slice thickness of $3 \mathrm{~mm}$ (Fig. 3a-c) which included a first sequence (TR 600; TE 14; flip angle 15; band width 150) and a second sequence (TR 2,260, TE 14, flip angle 15, band width 150). We selected a matrix of $512 \times 512$ pixels combined with a field of view of $500 \mathrm{~mm}$. The T1w-TSE sequence has been established to produce best imaging results for implants and the least amount of intrinsic artifacting [4, 7, 10, 12, 14, 16, 25].

Using a current version of DICOM reader software, one author (TE) measured the artifact area on the scan of each of the nine implants six times, i.e., a total of 54 individual tracings were recorded and analyzed. The measurements started with the slice with the first artifacting reaction and ended with the last slice exhibiting an artifact reaction. Corresponding to the respective implant CSA, the middle slice of all slices exhibiting artifact reactions was defined as the MAA for each implant. To calculate the total artifact volume (TAV) for each spacer, all artifact areas measured for that spacer were added and multiplied by the slice thickness of $3 \mathrm{~mm}$ according to the multisection slice technique described by Debatin [2]. The ratio of CSA to MAA and the ratio of IV to TAV were calculated and presented in tables (Table 1).

\section{Statistical analysis}

Newman-Keuls multiple comparisons were used to calculate intragroup differences in TAV and MAA (Table 2). $t$ test correlations were performed to determine any intergroup differences regarding the implant materials
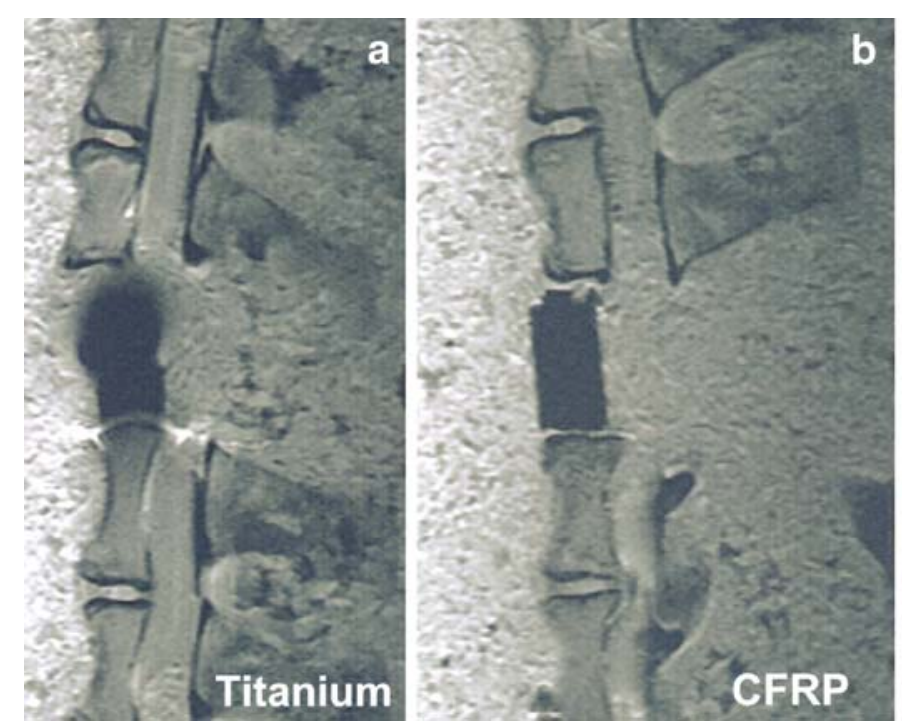

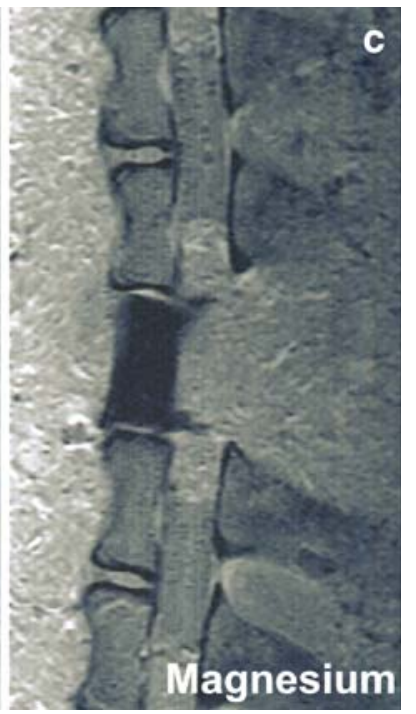


Table 2 Intragroup comparison of target variables.

\begin{tabular}{|c|c|c|c|c|c|}
\hline Spacer material & Size & $\mathrm{MAA}^{\mathrm{a}}, \mathrm{cm}^{2}($ mean $\pm \mathrm{SD})$ & Ratio CSA to MAA & $\mathrm{TAV}^{\mathrm{a}}, \mathrm{cm}^{2}($ mean $\pm \mathrm{SD})$ & Ratio IV to TAV \\
\hline \multirow[t]{3}{*}{ Group I MgAlMn50 $(n=3)$} & Small & $1.91 \pm 0.04$ & $1: 1.3$ & $1.83 \pm 0.09$ & $1: 1.5$ \\
\hline & Medium & $3.26 \pm 0.06$ & $1: 1.4$ & $4.17 \pm 0.09$ & $1: 1.8$ \\
\hline & Large & $4.06 \pm 0.07$ & $1: 1.2$ & $5.08 \pm 0.15$ & $1: 1.3$ \\
\hline \multirow[t]{3}{*}{ Group II TiAl6V4 $(n=3)$} & Small & $3.26 \pm 0.04$ & $1: 2.2$ & $5.71 \pm 0.09$ & $1: 4.8$ \\
\hline & Medium & $4.61 \pm 0.23$ & $1: 1.9$ & $9.32 \pm 0.10$ & $1: 4.1$ \\
\hline & Large & $5.54 \pm 0.04$ & $1: 1.6$ & $10.84 \pm 0.13$ & $1: 2.9$ \\
\hline \multirow[t]{3}{*}{ Group III CFRP $(n=3)$} & Small & $1.89 \pm 0.07$ & $1: 1.3$ & $1.81 \pm 0.07$ & $1: 1.5$ \\
\hline & Medium & $3.18 \pm 0.06$ & $1: 1.3$ & $4.09 \pm 0.11$ & $1: 1.7$ \\
\hline & Large & $4.06 \pm 0.13$ & $1: 1.2$ & $5.08 \pm 0.13$ & $1: 1.3$ \\
\hline
\end{tabular}

$C S A$ cross-sectional area, MAA median artifact area, $I V$ implant volume, $T A V$ total artifact volume, $S D$ standard deviation

${ }^{\text {a }}$ Newman-Keuls multiple-comparison analysis $p<0.001$

(Table 2). A $p$ value $<0.05$ indicated a significant difference between the means of any two groups.

\section{Results}

Table 1 presents the spacer dimensions. Table 2 shows the intragroup comparisons of target variables. Table 3 lists the results of the intergroup $t$ test correlations between TAV and MAA in relation to spacer material. Mean artifacting behavior increased with spacer size. When magnesium was compared with titanium, there were significant differences in both MAA and TAV. When magnesium was compared with carbon, the differences were not significant. In fact, magnesium produces an artifacting behavior very similar to that of CFRP.

\section{Discussion}

Spinal surgeons have not stopped searching for the optimum spacer material that combines high biocompatibility with artifact-free MRI imaging behavior in the

Table 3 Intergroup comparisons of artifacting behavior by $t$ test correlation.

\begin{tabular}{llcc}
\hline Spacer material & Size & MAA $P$ value $^{\mathrm{a}}$ & TAV $P$ value \\
\hline Group I vs. group II & Small & $\leq 0.001$ & $\leq 0.001$ \\
& Medium & $\leq 0.001$ & $\leq 0.001$ \\
& Large & $\leq 0.001$ & $\leq 0.001$ \\
Group I vs. group III & Small & 0.59 & 0.61 \\
& Medium & 0.09 & 0.26 \\
& Large & 1.0 & 0.96 \\
\hline
\end{tabular}

$M A A$ median artifact area, $T A V$ total artifact volume

${ }^{\text {a }}$ Significance level $p<0.05$ implant environment. This study was conducted to determine whether cylindrical spacers made of the biomaterial magnesium are suitable as spinal implants by comparing their MRI artifacting with that of identically dimensioned spacers made of a titanium alloy and a carbon-fiberreinforced polymer.

In radiological spinal diagnostics, MRI is highly effective for clarifying postfusion questions regarding osseus and soft tissue structures in relation to implant position. A comparative in vitro study shows that MRI has a higher sensitivity than $\mathrm{CT}$ in detecting osseus changes in the implant's direct surroundings [24]. Moreover, MRI is well suited to demonstrate myelopathies, inflammatory and infectious processes, and any neurodegenerative changes. The MRI imaging behavior of metallic spinal implants is obviously well documented in the literature $[6,10,13-16$, $19,22,23,25]$. However, the aims of the published studies differed in that most focused on determining sequencerelated artifact size. In a comparative cadaveric artifact studies, Wang et al. [26] described the MRI behavior of intervertebral spacers made of titanium and tantalum. The authors concluded that T1- and T2-weighted spin echo images were suitable for both metals to visualize the neural structures of the spine with comparable amounts of artifact. The artifact rate of the titanium spacer as well as the tantalum spacer was primarily limited to the implant's direct surroundings and anatomic neighboring structures were clearly distinguishable.

In a phantom study by Rudisch et al. [15], the relevance of metallic artifacts and implant-related characteristics, such as implant material and position, was demonstrated in addition to effects caused by the selected MRI sequence. In materials with a higher magnetizability like titanium alloys, implant shape additionally has an effect on the range of MRI artifacts [5].

The results of this comparative study showed that implant material and volume both affected the MRI 
artifacting behavior of our cylindrical test spacers. It was also noted that the smaller the implant size, the smaller was the range of susceptibility artifacts produced. The ratios calculated in Table 2 prove that the magnesium metal alloy exhibited behavior artifacting that was more like a nonmetal. In this context, the magnesium test spacer produced significantly less artifact compared to the titanium implant. Given its osseoconductive potential as a metal [9, 18], implant alloys made with magnesium would combine the advantages to the two principal spacer materials currently used, but without their limitations, at least in terms of MRI artifacting

Acknowledgements The authors thank Dr. Mark Riner of MedTech Composites GmbH, Switzerland, the Peter Brehm Company, Weisendorf, Germany, and the Material Science at the Technical University of Hannover, Germany for producing the respective test implants.

Conflict of interest statement We declare that we have no conflict of interest.

\section{References}

1. Brantigan JW, Steffee AD (1993) A carbon fiber implant to aid interbody lumbar fusion. Two-year clinical results in the first 26 patients. Spine 18:2106-2107

2. Debatin JF, Nadel SN, Sostman HD, Spritzer CE, Evans AJ, Grist TM (1992) Magnetic resonance imaging-cardiac ejection fraction measurements. Phantom study comparing four different methods. Invest Radiol 27:198-204. doi:10.1097/00004424-19920300000003

3. Diedrich O, Kraft CN, Perlick L, Schmitt O (2001) The posterior lumbar interbody fusion with cages (PLIF) and transpedicular stabilization. Zentralbl Neurochir 62:106-113. doi:10.1055/s-200121796

4. Ernstberger T, Heidrich G, Bruening T, Krefft S, Buchhorn G, Klinger HM (2006) The interobserver-validated relevance of intervertebral spacer materials in MRI artifacting. Eur Spine J $7: 1-7$

5. Ernstberger T, Heidrich G, Buchhorn G (2007) Post implantation MRI with cylindric and cubic intervertebral test implants: evaluation of implant shape, material and volume in MRI artifacting - an in vitro study. Spine J 7:353-359. doi:10.1016/j.spinee.2006.03.016

6. Ernstberger T, Heidrich G, Schultz W, Grabbe E (2007) Implant detectability of intervertebral disc spacers in post fusion MRI: evaluation of the MRI scan quality by using a scoring system-an in vitro study. Neuroradiology 49:103-109. doi:10.1007/s00234006-0161-5

7. Henk CB, Brodner W, Grampp S, Breitenseher M, Thurnher M, Mostbeck GH, Imhof H (1999) The postoperative spine. Top Magn Reson Imaging 10:247-264. doi:10.1097/00002142-19990800000006

8. Kuklo TR, Potter BK, Bell RS, Moquin RR, Rosner MK (2006) Single-stage treatment of pyogenic spinal infection with titanium mesh cages. J Spinal Disord Tech 19:376-382. doi:10.1097/01. bsd.0000203945.03922.f6

9. Lambotte A (1932) L'utilisation du magnesium comme materiel perdu dans l'ostheosynthèse. Bull Mem Coc Nat Chir 28:13251334
10. Malik AS, Boyko O, Atkar N, Young WF (2001) A comparative study of MR imaging profile of titanium pedicle screws. Acta Radiol 42:291-293. doi:10.1080/028418501127346846

11. McAfee PC, DeVine JG, Chaput CD, Prybis BG, Fedder IL, Cunningham BW, Farrell DJ, Hess SJ, Vigna FE (2005) The indications for interbody fusion cages in the treatment of spondylolisthesis: analysis of 120 cases. Spine 30(Suppl 6):S60S65

12. Ortiz O, Pait TG, McAllister P, Sauter K (1996) Postoperative magnetic resonance imaging with titanium implants of the thoracic and lumbar spine. Neurosurgery 38:741-745. doi:10.1097/ 00006123-199604000-00022

13. Petersilge CA, Lewin JS, Duerk JL, Yoo JU, Ghaneyem AJ (1996) Optimizing imaging parameters for MR evaluation of the spine with titanium pedicle screws. Am J Roentenol 166:1213-1218

14. Rauzzino MJ, Shaffrey CI, Nockels RP, Wiggins GC, Rock J, Wagner J (1999) Anterior lumbar fusion with titanium threaded and mesh interbody cages. Neurosurg Focus 15:e7

15. Rudisch A, Kremser C, Peer S, Kathrein A, Judmaier W, Daniaux H (1998) Metallic artifacts in magnetic resonance imaging of patients with spinal fusion. A comparison of implant materials and implant sequences. Spine 23:692-699. doi:10.1097/00007632-19980315000009

16. Rupp R, Ebraheim NA, Savolaine ER, Jackson WT (1993) Magnetic resonance imaging evaluation of the spine with metal implants. General safety and superior imaging with titanium. Spine 18:379-385

17. Schreiner U, Schwarz M, Scheller G, Schroeder-Boersch H, Jani L (2000) Knöchernes Einwachsverhalten von Probekörpern aus kohlefaserverstärktem Kunststoff. Z Orthop Ihre Grenzgeb 138:540-543. doi:10.1055/s-2000-9598

18. Staiger MP, Pietak AM, Huadmai J, Dias G (2006) Magnesium and its alloys as orthopaedic biomaterials: a review. Biomat 27:1728-1734. doi:10.1016/j.biomaterials.2005.10.003

19. Thomsen M, Schneider U, Breusch SJ, Hansmann J, Freund M (2001) Artefacts and ferromagnetism dependent on different metal alloys in magnetic resonance imaging. An experimental study. Orthopade 30:540-544. doi:10.1007/s001320170063

20. Troitskii VV, Tsitrin DN (1944) The resorbing metallic alloy 'Ostheosinthzit' as material for fastening broken bone. Khirurgiia 8:41-44

21. Trouillier H, Birkenmaier C, Rauch A, Weiler C, Kauschke T, Refior HJ (2006) Posterior lumbar interbody fusion (PLIF) with cages and local bone graft in the treatment of spinal stenosis. Acta Orthop Belg 72:460-466

22. Vaccaro AR, Chesnut RM, Scuderi G, Healy JF, Massie JB, Garfin SR (1994) Metallic spinal artifacts in magnetic resonance imaging. Spine 19:1237-1242

23. Van Goethem JW, Parizel PM, Jinkins JR (2002) Review article: MRI of the postoperative lumbar spine. Neuroradiology 44:723739. doi:10.1007/s00234-002-0790-2

24. Walde TA, Weiland DE, Leung SB, Sychterz CJ, Ho S, Engh CA, Potter HG (2005) Comparison of CT, MRI and radiographs in assessing pelvic osteolysis: a cadaveric study. Clin Orthop Relat Res 437:138-144. doi:10.1097/01.blo.0000164028.14504.46

25. Wang JC, Sandhu HS, Yu MD, Minchew JT, Delamarter RB (1997) MR parameters for imaging titanium spinal instrumentation. J Spinal Disord 10:27-32. doi:10.1097/00002517-199702000-00004

26. Wang JC, Yu WD, Sandhu HS, Tam V, Delamarter RB (1998) A comparison of magnetic resonance and computed tomographic image quality after the implantation of tantalum and titanium spinal instrumentation. Spine 1:1684-1688. doi:10.1097/00007632199808010-00014

27. Znamenskii MS (1945) Metallic osteosynthesis by means of an apparatus made of resorbing metal. Khirurgiia 12:60-63 